\title{
Forecasting Visitors' behaviour in Crowded Museums
}

\author{
Caterina Balzotti ${ }^{1}$, Maya Briani ${ }^{2}$, Alessandro Corbetta ${ }^{3}$, Emiliano Cristiani $^{2}$, \\ Marina Minozzi ${ }^{4}$, Roberto Natalini², Sara Suriano ${ }^{5}$, Federico Toschi ${ }^{2,3}$ \\ ${ }^{1}$ Dipartimento di Scienze di Base e Applicate per l'Ingegneria, Sapienza Università di Roma, Rome, Italy \\ ${ }^{2}$ Istituto per le Applicazioni del Calcolo, Consiglio Nazionale delle Ricerche, Rome, Italy \\ ${ }^{3}$ Department of Applied Physics, Eindhoven University of Technology, Eindhoven, The Netherlands \\ ${ }^{4}$ Galleria Borghese, Rome, Italy \\ ${ }^{5}$ Dipartimento di Matematica, Sapienza Università di Roma, Rome, Italy
}

\begin{abstract}
In this paper we tackle the issue of measuring and understanding the visitors' dynamics in a crowded museum in order to create and calibrate a predictive mathematical model. The model is then used as a tool to manage, control and optimize the fruition of the museum. Our contribution comes with one successful use case, the Galleria Borghese in Rome, Italy.
\end{abstract}

Keywords: pedestrian modelling, complex behaviour, floor usage, data acquisition, museums.

\section{Introduction}

Operating public facilities such as museums, which come as intricate assemblies of rooms, entails a complex technological issue: the optimization of the floor usage. This aims at ensuring that visitors' flows develop safely and comfort levels are maintained at all times. Overall, a successful floor usage optimization demands different capabilities, namely monitoring, forecasting, and, ultimately, steering the visitors' dynamics.

Monitoring and forecasting involve a mixture of challenges: the first demands continuous and reliable data acquisition, the second requires complexity reduction, modelling and the understanding of some psychological aspects of crowd motion.

In this contribution we tackle the issue of forecasting the visitors' dynamics and create a predictive mathematical model for the visitors' behaviour in a museum. Our model is aimed at enhancing the guests' experience, and particularly at preventing under- and over-occupied areas.

\section{The 'Galleria Borghese' in Rome}

Our contribution comes with one successful use case: the optimization of the visitors' dynamics at Galleria Borghese in Rome, Italy. Galleria Borghese is one of the most famous museums in the world, unique with regard to Bernini's sculptures and Caravaggio's paintings. The museum receives more than 500,000 visitors per year, which is quite a large number considering the small size of the exhibition space.

The museum has several historical, artistic and architectural constraints which limit its usability. In addition, the two floors of the museum have different capacity limits (because of the different escape routes), and the artworks cannot be exposed to excessive temperature and humidity variations.

Currently, the visits are organized as follows. Tickets must be booked in advance, and two hours are given to stay in the museum. A small percentage of tickets, called "last minute", are instead sold at the moment. At the end of each period of visit, people are invited to leave, and the museum empties completely. Visits are not organized - on purpose - through an obligatory exhibition path (i.e. a predetermined sequence of rooms). Moreover, the circular structure of the building makes the visit not a one-way route: visitors tend to come back several times to the same rooms (the density of the artworks is so high that one can miss completely some pieces on the first visit) and some effort must be done to find the stairway which connects the lower with the upper level. 
The main goal of this research is to increase the number of visitors per day while keeping the number of people within the limits and under an acceptable comfort level at any time. The control variables of the museum are the maximum number of sold tickets, the opening hours, the maximum duration of the visit, the maximum number of guided groups allowed at the same time, and others.

\section{Methods}

\subsection{Data acquisition}

Presence data were collected manually, simply counting the passages of people moving from one room to another in the whole museum. This was done for four turns of visit during four days, by means of a specifically designed smartphone app. The app, connected to a database, was able to record the data (including the direction of the movement) and its timestamp in real time. Such measurements allowed us to get the number of people in each room at each time. The duration of the visit of each room was also measured from a sample of visitors (about 500).

\subsection{Mathematical model}

The complex behaviour of visitors inside the museum makes the prediction of the path of each single visitor almost impossible. On the contrary, it is feasible to predict the movements of the visitors as a whole. For this reason, although our model treats the visitors' motion individually, we do not consider the problem of tracking single persons. Rather, we adopt an "Eulerian" point of view, being interested only in the number of people present in every room at all times, and the flows of people through museum's doors. The visitors' dynamics deploy over an undirected graph representing the museum rooms, see Fig. 1. Thus, while each room is simplified as a graph node, connections among different rooms, such as doors or staircases, define the edges of the graph.

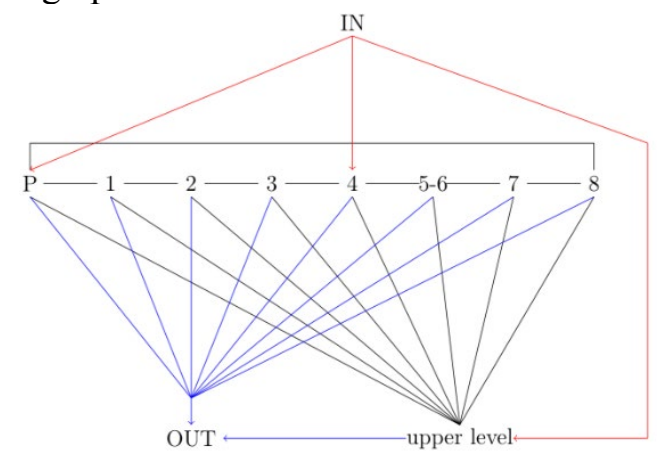

Fig. 1: Graph representing the connections among the rooms of the museum. Numbers 1-8 denote the eight rooms of the lower level, ' $\mathrm{P}$ ' is the entrance hall. The museum has three access points (red lines). For simplicity, all rooms at the upper level are unified in a single node and we assume that exit and upper level can be accessed by any room.

Initially visitors are placed in a waiting room (IN, cf. Fig. 1). The time spent in this room is computed accordingly to a specific probability distribution function, in order to take into account that people do not all enter together (because ticketing requires a few seconds) and that some people arrive late. Once the visitor is in a room, the algorithm assigns the room visit time (RVT), i.e. the desired duration of the stay in that room, sampling a normally distributed random variable shaped in accordance to measured data. The RVT is then modified based on the current degree of congestion of the room (slight congestion increases the RVT, high congestion decreases it) and based on the number of times that room was already visited (known rooms are visited more rapidly, and no room can be visited more than three times). Once the visit of the room is completed, the visitor moves to another room. Among the connected rooms, the choice is random, with higher probability to move to yet unvisited or less visited locations. The probability to change floor, instead, is proportional to the number of visited rooms in the current floor 
(but the upper level can be visited only once). When the whole museum is visited or the time allowed for visit is over, the visitor leaves the museum (moves to OUT, cf. Fig. 1).

A calibration of the model parameters allowed us to get the result shown in Fig. 2: The model is able to reproduce nicely the occupancy over time, both in the whole museum and in the two floors separately.
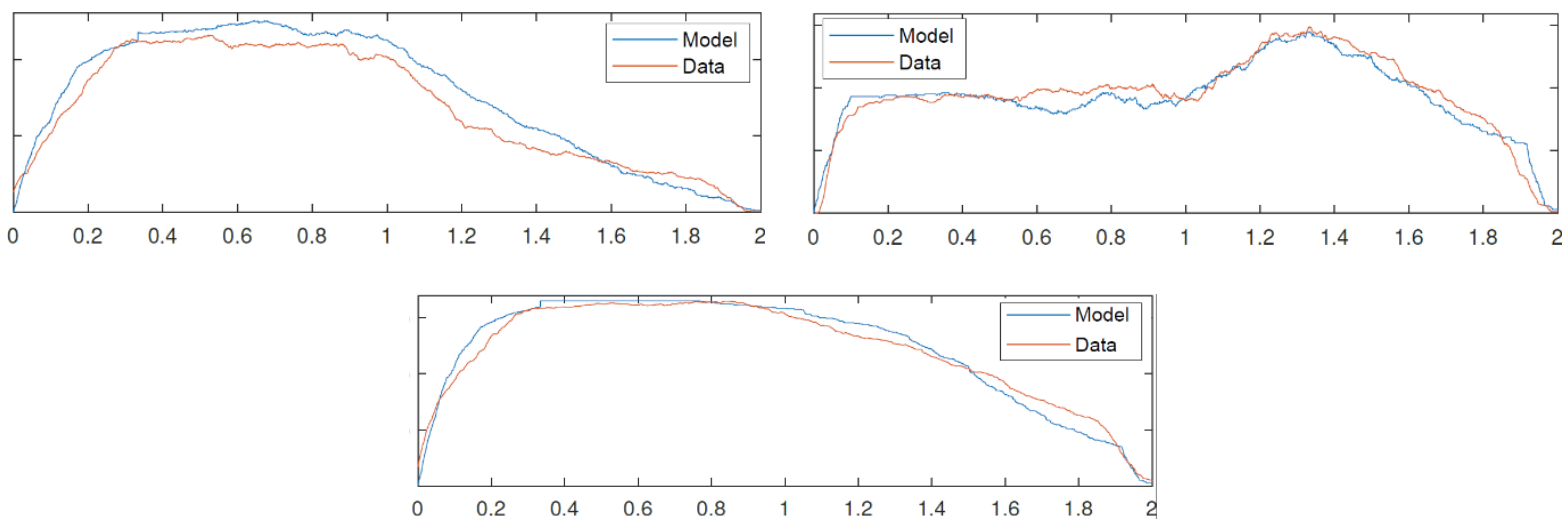

Fig. 2: Profile of the number of visitors as a function of time (0-2h, i.e. a single turn) computed by the model and compared with measured data (top-left: lower level, top-right: upper level, bottom: whole museum). Exact numbers are not made public by the museum.

\section{Results}

In this section we report the results of the simulations corresponding to three scenarios, see Fig. 3. In the first one we reproduce the strategy currently employed in the museum. Every $2 \mathrm{~h}$ (from 9 a.m. to 7 p.m.) 360 visitors enter the museum, 270 of them begin the visit from the lower level, while 90 begin from the upper level. At the end of the turn, visitors are invited to leave.

In the second scenario, we test the same entrance strategy $(270+90)$ but we drop the forced exit, i.e. people are free to stay more than two hours. As it can be seen, the overall occupancy is not larger than the first scenario. This is plausible since we observed that two hours are enough for most people and, in the first scenario, about 5 minutes are lost in emptying and re-filling the museum again.

In the third scenario, the strategy resulted from a gradient-based optimization algorithm. The goal was to maximize the number of visitors per day under two constraints: the maximum number of visitors allowed in the lower level and in the upper level. The two control variables were the number of people beginning the visit from the lower and the upper level respectively. Moreover, people enter every hour, with no forced exit after two hours. Initially, 140+90 people enter the museum, then $190+0$ people enter every hour until the final turn, when $250+30$ people go in. In this way we are able to reduce significantly the congested period at the upper level (see Fig. 2 top-right, around 1.3h), to avoid dead periods and, most important, increasing by 40 the total number of visitors per day.
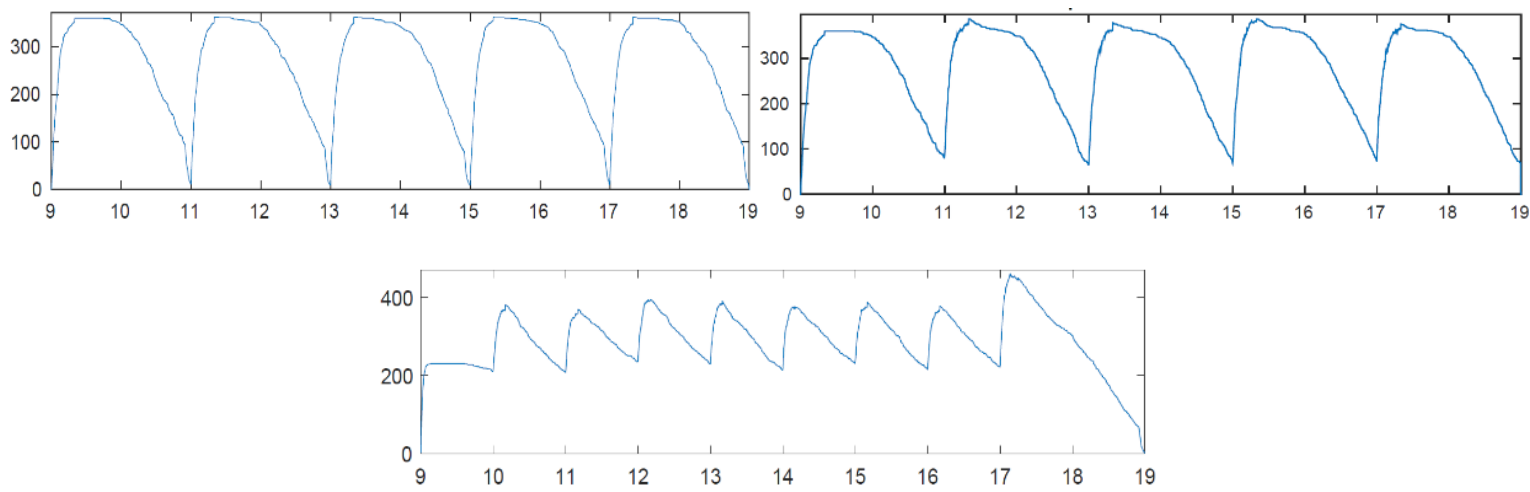

Fig. 3: Number of visitors in the whole museum as a function of the hour of the day (top-left: first scenario, top-right: second scenario, bottom: third scenario). In the second and third scenario the museum is never empty. 